\title{
Lactate-guided resuscitation saves lives: we are not sure
}

\author{
Jan Bakker ${ }^{1,2,3^{*}}$, Daniel de Backer ${ }^{4,5}$ and Glenn Hernandez ${ }^{2}$
}

(C) 2016 Springer-Verlag Berlin Heidelberg and ESICM

A fundamental objective of septic shock resuscitation is rapid restoration of tissue perfusion. However, one of the most crucial unresolved challenges is to identify a clinical physiological variable that closely reflects global or regional hypoperfusion, or cellular hypoxia, and thus could be potentially used as an accurate resuscitation target.

Since the first description of lactate levels in humans, hyperlactatemia has been traditionally considered as a signal of tissue hypoxia. Both experimental and clinical studies have shown that a reduction in global oxygen delivery will ultimately result in a decrease in oxygen consumption. When oxygen demand remains stable, this decrease in oxygen consumption hallmarks the occurrence of tissue hypoxia and is associated with a sharp increase in lactate levels $[1,2]$.

However as lactate is a normal product of glucose metabolism, many other factors could increase lactate levels even in the presence of adequate oxygen supply. Probably the most confounding factor is adrenergicdriven aerobic glycolysis triggered by stress situations. Circulatory failure is associated with significant sympathetic activation resulting in increased muscle release of lactate as a systemic metabolic fuel [3]. Thus, persistent hyperlactatemia could simply be a marker of the severity of shock or stress rather than reflecting real impairment of tissue perfusion/oxygenation.

Other factors, particularly abnormal lactate clearance, may also contribute to hyperlactatemia in the presence of adequate perfusion, although the evidence is not uniform.

\footnotetext{
*Correspondence: jan.bakker@erasmusmc.nl

${ }^{1}$ Department Intensive Care Adults, Erasmus MC University Medical Center, Room H625, P.O. Box 2040, 3000 CA Rotterdam, The Netherlands Full list of author information is available at the end of the article
}

Contrasting viewpoints can be found at: doi:10.1007/s00134-015-4196-0 and doi:10.1007/s00134-016-4235-5.
Whereas Levraut et al. [4] demonstrated that impaired clearance was a significant cause of mild hyperlactatemia in stable septic patients, Revelly et al. [5] demonstrated, with stable isotopes, that lactate clearance was not the main cause of hyperlactatemia. More recently, Tapia et al. [6] showed an almost negligible exogenous lactate clearance in early experimental septic shock despite preserved liver perfusion and a concomitant normal clearance of the alcohol sugar sorbitol.

In summary, persistent hyperlactatemia may represent a state of physiological disequilibrium between increased production (either aerobic or anaerobic) and impaired clearance. In this sense, pursuing lactate normalization through further resuscitation with fluids or inotropes when other signs of tissue hypoperfusion have disappeared may expose the patient to the toxicity of overresuscitation without any clear benefit. This highlights one of the major dilemmas during shock resuscitation: when to consider that a persistent hyperlactatemia is still the consequence of inadequate perfusion. A couple of algorithms to address this question have been proposed and are based on multimodal perfusion monitoring. Persistent hyperlactatemia but with normal central venous oxygen saturation $\left(\mathrm{ScvO}_{2}\right)$, central venous-arterial $\mathrm{pCO}_{2}$ gradient $\left[\mathrm{P}(\mathrm{cv}-\mathrm{a}) \mathrm{CO}_{2}\right]$, and peripheral perfusion may indicate a lower probability of residual hypoperfusion, although this needs to be confirmed by future studies.

In support of this idea, a recent study [7] suggested that the time course of lactate normalization during a successful resuscitation follows a biphasic curve: an early rapid response (a flow-responsive phase) followed by a later slower recovery trend potentially explained by non-flow-dependent mechanisms (Fig. 1). Interestingly, some flow-responsive variables such as $\mathrm{ScvO}_{2}, \mathrm{P}(\mathrm{cv}-\mathrm{a})$ $\mathrm{CO}_{2}$, and capillary refill time (CRT) exhibited much higher normalization rates at $6 \mathrm{~h}$ than lactate. Only half of this cohort of survivors normalized lactate at $24 \mathrm{~h}$.

\section{Springer}




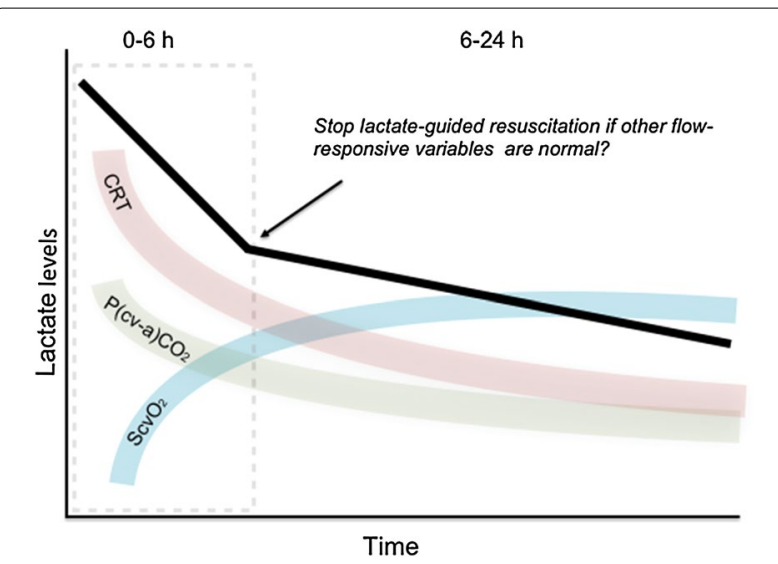

Fig. 1 Theoretical recovery time-course of hyperlactatemia in septic shock survivors shows a biphasic curve with an early rapid decrease associated with normalization of other flow-responsive variables, and a much slower recovery trend after this initial phase, a fact potentially explained by non-flow-dependent mechanisms (adapted from [7]). CRT capillary refill time, $P(\mathrm{CV}-a) \mathrm{CO}_{2}$ central venous to arterial $\mathrm{pCO}_{2}$ gradient, $\mathrm{ScVO}_{2}$ central venous $\mathrm{O}_{2}$ saturation

Admittedly, other series found slower normalization of some of these variables, which highlights the interest of multimodal monitoring.

Some data tend to support that lactate-guided resuscitation strategies should be associated with multimodal perfusion monitoring and be focused on the early phase. De Backer et al. demonstrated that early improvement of microcirculatory flow with dobutamine was associated with rapid decrease in lactate [8]. A recently published therapeutic algorithm focused on lactate-driven resuscitation exclusively in the first $8 \mathrm{~h}$ of ICU management with a significant favorable impact on outcome [9]. A recent meta-analysis also showed that early resuscitation was associated with improved outcome, whereas late treatment was not [10].

To add to the complexity, an important finding in the study by Jansen et al. [9] was that the time course of lactate levels was identical in both the protocol and control group, although the treatment team had no access to lactate levels in the control group. The differences in hemodynamic management between the two groups were minor although in agreement with the pivotal study on early goal-directed therapy: more intensive treatment in the study period and less in the follow-up period for the protocol patients when compared to the control group patients. Although the survival was significantly better in the protocol group this was not reflected by the changes in lactate levels, either in the study period (first $8 \mathrm{~h}$ ) or in the follow-up period (up to $72 \mathrm{~h}$ following inclusion). In addition, van Genderen et al. [11] recently showed that limiting fluid resuscitation in patients with a persistent clinical problem (increased lactate levels, low urine output, persisting hypotension, etc.) but with normal peripheral perfusion was safe and associated with an improvement in organ function. These findings raise some doubts over the whole conception of lactate-guided resuscitation.

Consequently a general recommendation to target the circulation in patients with increased lactate levels is too simplistic and not sufficiently supported by clinical studies. It seems reasonable to optimize systemic hemodynamics and microcirculation early in the phase of septic shock when there is a clinical problem and markers of microcirculatory perfusion are abnormal. In this phase several flow-responsive variables will probably improve in parallel. After this initial resuscitation, persistently elevated lactate levels probably reflect an ongoing septic process, hyperadrenergia, or metabolic deterioration, especially if the other perfusion variables are normal.

In the meantime, whenever lactate levels rise in a septic patient, the patient is at increased risk of morbidity and mortality. This first requires a thorough investigation into the likely causes of this hyperlactatemia. When abnormal tissue perfusion or oxygenation is likely involved, optimizing global hemodynamics and microcirculation for a short period of time is associated with improved outcome.

\section{Author details \\ ${ }^{1}$ Department Intensive Care Adults, Erasmus MC University Medical Center, Room H625, P.O. Box 2040, 3000 CA Rotterdam, The Netherlands. ${ }^{2}$ Departa- mento de Medicina Intensiva, Facultad de Medicina, Pontificia Universidad Católica de Chile, Santiago, Chile. ${ }^{3}$ Division of Pulmonary, Allergy, and Critical Care Medicine, Department of Medicine, Columbia University Medical Center, New York, USA. ${ }^{4}$ Université Libre de Bruxelles, Brussels, Belgium. ${ }^{5}$ Department of Intensive Care, CHIREC Hospitals, Brussels, Belgium.}

\section{Compliance with ethical standards}

\section{Conflicts of interest}

The authors have no conflict of interest regarding this manuscript.

Received: 8 January 2016 Accepted: 9 January 2016

Published online: 1 February 2016

\section{References}

1. Jansen TC, van Bommel J, Bakker J (2009) Blood lactate monitoring in critically ill patients: a systematic health technology assessment. Crit Care Med 37:2827-2839

2. Friedman G, De Backer D, Shahla M, Vincent JL (1998) Oxygen supply dependency can characterize septic shock. Intensive Care Med 24:118-123

3. Bakker J, Nijsten MW, Jansen TC (2013) Clinical use of lactate monitoring in critically ill patients. Ann Intensive Care 3:12

4. Levraut J, Ciebiera JP, Chave S, Rabary O, Jambou P, Carles M, Grimaud D (1998) Mild hyperlactatemia in stable septic patients is due to impaired lactate clearance rather than overproduction. Am J Respir Crit Care Med 157:1021-1026 
5. Revelly JP, Tappy L, Martinez A, Bollmann M, Cayeux MC, Berger MM, Chiolero RL (2005) Lactate and glucose metabolism in severe sepsis and cardiogenic shock. Crit Care Med 33:2235-2240

6. Tapia P, Soto D, Bruhn A, Alegria L, Jarufe N, Luengo C, Kattan E, Regueira T, Meissner A, Menchaca R, Vives MI, Echeverria N, Ospina-Tascon G, Bakker J, Hernandez G (2015) Impairment of exogenous lactate clearance in experimental hyperdynamic septic shock is not related to total liver hypoperfusion. Crit Care 19:188

7. Hernandez G, Luengo C, Bruhn A, Kattan E, Friedman G, Ospina-Tascon GA, Fuentealba A, Castro R, Regueira T, Romero C, Ince C, Bakker J (2014) When to stop septic shock resuscitation: clues from a dynamic perfusion monitoring. Ann Intensive Care 4:30

8. De Backer D, Creteur J, Dubois MJ, Sakr Y, Koch M, Verdant C, Vincent $J \mathrm{~L}$ (2006) The effects of dobutamine on microcirculatory alterations in patients with septic shock are independent of its systemic effects. Crit Care Med 34:403-408

9. Jansen TC, van Bommel J, Schoonderbeek FJ, Sleeswijk Visser SJ, van der Klooster JM, Lima AP, Willemsen SP, Bakker J (2010) Early lactate-guided therapy in intensive care unit patients: a multicenter, open-label, randomized controlled trial. Am J Respir Crit Care Med 182:752-761

10. Gu WJ, Wang F, Bakker J, Tang L, Liu JC (2014) The effect of goal-directed therapy on mortality in patients with sepsis - earlier is better: a metaanalysis of randomized controlled trials. Crit Care 18:570

11. van Genderen ME, Engels N, van der Valk RJP, Lima A, Klijn E, Bakker J, van Bommel J (2015) Early peripheral perfusion-guided fluid therapy in patients with septic shock. Am J Respir Crit Care Med 191:477-480 